\title{
Thyroid Lesions - Cytodiagnosis by Fine Needle Aspiration and Non-Aspiration Cytology - Study of 3934 Cases
}

\author{
Dr: K.A Raju, \\ Associate Professor Department of Pathology Government Medical College, Palakkad
}

\begin{abstract}
All thyroid nodules are not cancer and the fine needle aspiration cytology is an accepted cost effective simple method for screening the nodules for malignancy. A three-year study of thyroid lesions (March 2012 to march 2015) with aspiration and non-aspiration cytology for each. The main purpose of the study was to detect the prevalence of carcinoma by cytology and quality assessment of non-aspiration over aspiration cytology. The main findings of the study are non-aspiration technique is diagnostically much superior than aspiration techniques. Prevalence of carcinoma by cytology is $5.6 \%$.
\end{abstract}

Keywords:Aspiration, Cytology, Diagnosis,Lesions Non- aspiration, Thyroid,

\section{INTRODUCTION}

Thyroid swellings are unique in the sense that there are no definite criteria by which one can predict the behaviour and survivalrate. Knowledge of the prognosis of thyroid diseases, in general depends on the exact histological diagnosis and adequate follow up.

All thyroid nodules are not cancer and the Fine Needle Aspiration Cytology (FNAC) is an accepted, cost effective, simple method for screening the nodules for malignancy. Rapid diagnosis, costeffectiveness, greater patient acceptance, repeatability and the ability to sample a tissue throughout most of its volume are among the noteworthy merits of FNAC.This diagnostic modality involves firm attachment of the aspirating needle to a syringe, usually held in a specially designed syringe holder, which enables single handed strong suction to be applied. Particularly vascular organs such as thyroid or haemorrhagic tumours, this technique is not infrequently complicated by aspiration of significant quantities of fresh blood, which compromises cellularconcentration, preservationand interpretation.

In an attempt to overcome this problem, an alternative fine needle sampling method developed in France was further investigated. This technique employing insertion of a fine needle into lesion without attachment of a syringe, relies on the property of capillary tension in narrow channels (SandosandLeiman 1988). This principle of physics states that a fluid or semifluid substance will ascend spontaneously into a narrow tube, in inverse proportion to the diameter of the tube. This method of non-aspiration fine needle cytology which utilise no action, suction or aspiration, has been called 'cytopuncture' by French authors or fine needle capillary(FNC) (Bukerand Mir 1956) sampling. It has been shown that in the fine needle capillary sample the cellular material was more concentrated, less traumatised and less lightly tobe obscured by blood or distorted within blood clots. Morphological details were thus improved, resulting in easier interpretation and shorter screening times. For the patients, least complaining of pain or discomfort, for an operator, the technique is much simpler eliminating the need for a syringe holder and the needle held in between the fingers act as a sensor or sensitive probe.In this respect, providing tactile information not yielding when syringes or syringe holders are employed.

For thyroid nodules, solitary nodules are a common presentation. In contrast malignancy is rare. The challenge is to pick out the few malignant cases for surgery and prevent intervention in the remaining. It is here that needle cytology find its indisputable application.

Other two uses of fine needle cytology are to avoid the confirmatory open biopsy in the case of anaplastic carcinomas and serve as therapeutic measure in the case of small cysts when swelling subside after aspiration without recurrence or residual swelling.

Several studies have shown that the greatest value of fine needle cytology lies in its potential ability to detect malignant lesions with high accuracy.

\section{OBJECTVES}

1. To screen the thyroid nodules by cytology to detect the prevalence of thyroid carcinomas.

2. To do an overall quality assessment of non-aspiration cytology over aspiration cytology in thyroid lesions. 


\section{MATERIALS AND METHODS}

The study was on the aspiration and non-aspiration or cytopunture cytology of different palpable thyroid lesions admitted in the surgical units of the Medical College Hospital, Kottayam, Kerala from September 2012 toMarch 2015.

The techniques involved aspiration of cellular material from the thyroid glands by means of a thin needle with an external diameter of 0.6 to $0.7 \mathrm{~mm}$. (21 to 23 gauge) attached to a syringe and without attachment to the syringe. The syringes used were $10 \mathrm{ml}$ disposable plastic syringes. The aspirate was prepared as smears for examination under the light microscope. The aspirate was examined grossly soon after aspiration and if found inadequate a repeat aspiration was done straight away.

\subsection{Fixation and Staining}

Half of the slides were wet fixed using ether -alcohol mixture and remainder were permitted to air dry. The alcohol fixed and air dried smears were stained with the Papanicolaou and Geimsa stains respectively.Subject to the availability of the smears, a few of them were stained by Haematoxylin and eosin. Both Papanicolaou and Geimsa smears gave satisfactory results as it brought out the cytological and the background details best. It was seen that Papanicolaou stained smears faded within 6 months of time but Geimsa stained smears did not fade even after two and a half years. The smears should remain in the $95 \%$ ethyl alcohol fixative for a minimum of 15 minutes prior to staining.

\subsection{Interpretation}

Two smears were interpreted. To categorically state whether a smear was benign or malignant was difficult. Hence the 'indeterminate' and 'suspicious' categories were introduced. The diagnostic term 'indeterminate ' was used synonymously with follicular neoplasms just to indicate that their benignancy or malignancycannot be commented upon. Theterm 'suspicious' was used for the borderline cases and malignant for the frankly atypical smears. For the purpose of study the suspicious category was merged with the malignant group.

\section{OBSERVATION}

A total number of 18,122 patients came for fine needle aspiration biopsy at Medical College Hospital, Kottayam from March 2012 to March 2015 and out of these 3934 patients were with the clinical diagnosis of various types of thyroid lesions. This comprises $21.71 \%$ (Table 1 ).

Table: 1. Year wise Distribution of Thyroid FNAC

\begin{tabular}{|l|l|l|l|}
\hline Period & Total FNAC & Thyroid FNAC & Percentage $(\boldsymbol{\%})$ \\
\hline $2012-2013$ & 5237 & 835 & 15.94 \\
\hline $2013-2014$ & 5469 & 1320 & 24.13 \\
\hline $2014-2015$ & 7416 & 1779 & 23.98 \\
\hline 3 years & 18122 & 3934 & 21.71 \\
\hline
\end{tabular}

Table II. The frequency of Fine Needle Aspiration finding from March 2012 to March 2015 i,e. of 3934 cases

\begin{tabular}{|l|l|l|l|l|l|l|}
\hline Diagnosis & $\mathbf{2 0 1 2}$ & $\mathbf{2 0 1 3}$ & $\mathbf{2 0 1 4}$ & $\mathbf{2 0 1 5}$ & Total & Percentage (\%) \\
\hline Thyroglossal cyst & 0 & 2 & 6 & 0 & 8 & 0.2 \\
\hline Colloid nodule & 193 & 292 & 371 & 94 & 950 & 24.15 \\
\hline Multinodular goitre & 236 & 575 & 822 & 258 & 1891 & 48.06 \\
\hline Toxic Goitre & & 4 & & 1 & 5 & 0.12 \\
\hline Adenomatous Goitre & 5 & 44 & 31 & 1 & 81 & 2 \\
\hline Dyshormonogenic goitre & & 1 & 1 & & 2 & \\
\hline Lymphocytic thyroiditis & 7 & 17 & 26 & 15 & 65 & 1.61 \\
\hline Granulomatous thyroiditis & & 2 & 5 & 2 & 9 & 0.22 \\
\hline Hashimoto's thyroiditis & & 6 & 3 & 1 & 10 & 0.25 \\
\hline
\end{tabular}


Thyroid Lesions - Cytodiagnosis By Fine Needle Aspiration And Non-Aspiration Cytology - Study...

\begin{tabular}{|l|l|l|l|l|l|l|} 
Follicular neoplasm & 116 & 133 & 81 & 8 & 338 & 8.38 \\
\hline Hurthle cell neoplasm & & 3 & 2 & 1 & 6 & 0.14 \\
\hline Papillary Carcinoma & 4 & 14 & 16 & 5 & 39 & 0.96 \\
\hline Medullary Carcinoma & & 1 & 3 & 2 & 6 & 0.14 \\
\hline Anaplastic Carcinoma & & 2 & 2 & 2 & 6 & 0.14 \\
\hline $\begin{array}{l}\text { Metastatic Squamous cell } \\
\text { carcinoma }\end{array}$ & & 2 & & 2 & 0.05 \\
\hline Total & 576 & 1242 & 1640 & 476 & 3934 & 100 \\
\hline $\begin{array}{l}\text { Sex distribution } \\
\text { Male Female }\end{array}$ & $\begin{array}{l}82 \\
494\end{array}$ & $\begin{array}{l}177 \\
1065\end{array}$ & $\begin{array}{l}234 \\
1406\end{array}$ & $\begin{array}{l}68 \\
408\end{array}$ & $\begin{array}{l}561 \\
3373\end{array}$ & 5 \\
\hline
\end{tabular}

\subsection{ClinicalPresentation}

The duration of the swelling varied from 1 month to 38 years. The mean duration was 3 years. The maximum incidence of benign lesions was observed in above 5 years'duration (68\%), whereas the maximum incidence of malignant lesion noted was 1-2 years' duration (46\%)

In most of the cases, right sided involvement was seen in this study.

Table III: Distribution of hyperplastic, inflammatory and neoplastic thyroid lesions

\begin{tabular}{|l|l|l|}
\hline \multicolumn{1}{|c|}{ Lesions } & \multicolumn{1}{c|}{ Number } & \multicolumn{1}{c|}{ Percentage (\%) } \\
\hline Colloid/hyperplastic lesions & 3459 & 87.85 \\
\hline Inflammatory lesions & 84 & 2.27 \\
\hline Neoplastic Lesions & 397 & 9.88 \\
\hline Total & 3934 & 100.00 \\
\hline
\end{tabular}

Out of the 3934 FNAC diagnosis during the 3 years $87.85 \%$ were of colloid or hyperplastic disorders which includes, colloidnodule, colloidgoitre, multinodular goitre toxic goitre,adenomatous goitre and dyshormonogenic goitre. Ofthese, multinodular goitres were of maximum number i.e,1891,48.06\% out of 3943, then colloid nodule, 950 i.e. $24.15 \%$.

Inflammatory disorders were of $2.27 \%$ which included Lymphocytic thyroiditis, Granulomatous thyroiditis and Hashimoto's thyroiditis. The maximum number of lesions belonged to Lymphocytic thyroiditis.

Regarding the follicular neoplasms, the generally held view is that follicular adenoma and follicular carcinoma cannot be distinguished cytologically, since invasiveness, the hallmark of malignancy will not be reflected in the cytology smears. So categorising all these tumours as 'follicular neoplasms' have been suggested.

Table: IV Incidence of Neoplastic Lesions by FNAC

\begin{tabular}{|c|c|c|c|}
\hline Neoplasm & Benign & Malignant & Total \\
\hline Hurthle Cell neoplasm & 6 & & \\
\hline Follicular neoplasm & 338 & & \\
\hline Papillary carcinoma & & 39 & \\
\hline Medullary carcinoma & & 6 & \\
\hline Anaplastic carcinoma & & 6 & \\
\hline Metastasis & & 53 & 397 \\
\hline Total & 344 & & \\
\hline
\end{tabular}


Hence the neoplastic lesions, including follicular neoplasms and Hurthle cell neoplasm along with other carcinomas constituted $9.88 \%$. Of these $85.13 \%$ were follicular neoplasms, $1.5 \%$ Hurthles cellneoplasms and $13.35 \%$ carcinomas excluding follicular carcinomas that were included along with follicular neoplasms.

Among the carcinomas, i.e, $13.35 \%$ of neoplastic lesions ,73\% were papillary carcinomas $11.3 \%$ each for medullary and anaplastic carcinomas and $3.4 \%$ metastasisingcarcinomas tothe thyroid.

To do an overall quality assessment of non-aspiration cytology over aspiration cytology, fine needle aspiration and non-aspiration cytology were done in random.

The stained smears were interpreted without the knowledge of the technique employed for any particular set of slides. Apart from the diagnosis, the comments were rendered as three qualitative categories.

1.Unsuitable smears: if the slides consisted of mainly blood or cellular material absent or inadequatefor determination of benign or malignant changes.

2. Diagnostic/Adequate: When it was possible to render an opinion on the nature of the lesion sampled, but when the cellular material present was suboptimal due to poor cellularity, simpledilution, degenerative changes or specimen entrapment in blood clots.

3. Diagnostic superior: If the cells or cellular aggregates were concentrated, wellpreserved, unobscured by background blood and excellently displayed, with retention of architectural patterns such as follicles, papillae and flat sheets.

The results of fine needle aspiration and non-aspiration procedures of 495 cases are summarised in table V

Table V Performance of Aspiration and Non-Aspiration Cytology of 495 Cases

\begin{tabular}{|l|l|l|l|l|}
\hline \multicolumn{1}{|c|}{ Result } & \multicolumn{1}{|c|}{ NAC } & Percentage $(\boldsymbol{\%})$ & \multicolumn{1}{|c|}{ FNAC } & \multicolumn{1}{c|}{ Percentage (\%) } \\
\hline Diagnostic superior & 159 & 32.12 & 21 & 4.24 \\
Diagnostic adequate & 318 & 64.24 & 372 & 5.15 \\
Unsuitable & 18 & 3.63 & 102 & 20.60 \\
\hline & 495 & 100 & 495 & 100 \\
\hline
\end{tabular}

Out of 495 cases that includes both neoplastic and non-neoplastic lesions of thyroid, diagnostically superior material was obtained in 159 cases $(32.12 \%)$ of the non- aspiration sample verses 21 cases (4.24\%) of aspiration sample. The difference is significant at the level of $p=0.0033$ by McNemar's test of significant change. If we add the diagnostically sufficient material to the diagnostic superior group, it is found that $96 \%$ of non-aspiration cytology materials are diagnostic versus $79 \%$ of FNAC materials.Unsuitable samples were obtained only in 18 cases $(3.63 \%)$ for NAC whereas for FNAC it was in 102 cases $(20,60 \%)$

Table.VI: Performance of NAC and FNAC in Non-neoplastic and Neoplastic Thyroid Nodules

\begin{tabular}{|c|c|c|c|c|c|c|c|c|}
\hline \multicolumn{5}{|l|}{ Non-Neoplastic } & \multicolumn{4}{|c|}{ Neoplastic } \\
\hline & NAC & $\%$ & FNAC & $\%$ & NAC & $\%$ & FNAC & $\%$ \\
\hline Diagnostic Superior & 108 & 29.26 & 15 & 4.06 & 51 & 40.47 & 6 & 4.76 \\
\hline Diagnostic & 243 & 5.85 & 273 & 73.98 & 75 & 59.52 & 99 & 78.57 \\
\hline \multirow[t]{2}{*}{ Unsuitable } & 18 & 4.87 & 81 & 21.95 & 0 & 0 & 21 & 16.66 \\
\hline & 369 & & 369 & 100 & 126 & & 126 & 100 \\
\hline
\end{tabular}

Out of 495 cases,369 lesions were non-neoplastic (74.5\%) and 126 neoplastic (25.5\%).

Lesions taken for FNAC and NAC are given below.

1.Non-neoplasticnodules included

(MNG,colloid goitre, colloid nodule):339

2. Inflammatory lesions(Supportive

thyroiditis Hashimoto's thyroiditisand others) : 30

3. Neoplastic nodules included $: 126$

(Follicular neoplasms : 98

Papillary carcinoma : $\quad 21$

Medullarary carcinoma : $\quad 4$

Anaplastic carcinoma : 3

Total : 126 
Of the non-neoplastic lesions, $91 \%$ were of colloid or hyperplastic nodules and of the neoplastic lesions, $77 \%$ were of follicular neoplasm.

Of the 369 non-aspiration cytology slides of non-neoplastic lesions, 351 (95.2\%) were diagnostic which included diagnostic superior 108 (29.02\%). But for FNAC slides 288 (78\%) were diagnostic which included diagnostic superior only 15 (4.06\%). The unsuitable sample obtained by NAC for non-neoplastic lesions were only $18(4.8 \%)$ but for FNAC, it was $81(21.95 \%)$. A similar trend was found for neoplastic nodules also regarding the diagnostic series, and rate of diagnostic superior slides were increased. Unsuitable slides were there from the FNAC series.

It was noted that for supportive lesions better cellular smear were obtained from aspiration techniques compared to non-aspiration techniques. Of 6lesions, all six were diagnostic superior for FNACs whereas for NACs, 3 diagnostic superior and 3 diagnostic.

\section{DISCUSSION}

The establishment of a tissue diagnosis is of paramount importance in the management of thyroid lesions. In a study done to assess the proportion of patients subjected to surgery, it was found that the percentage of patients operated on had decreased from 95\% to 60 without any significant decrease in malignant tumours.

The usual aspiration technique involves single handed strong suction to be applied. This technique is not infrequently complicated by aspiration of significant quantities of fresh blood which compromises cellular concentration, preservation and interpretation.

The non- aspiration technique of fine needle sampling without aspiration also called cytopunture or fine needle capillary sampling has been utilized in France but has not been widely publicised or promoted. There it was first used for breast lesions, later for orbital and periorbital tumours. Then the thyroid was selected, since it is a vascular organ that notoriously produces heavily blood stained aspirates. While the number of unsuitable specimens in this series were not statistically different with FNA ornon-aspiration needle sampling, the quality of the material studied was significantly superior in the non- aspiration samples. It is agreed that the presence of blood in thyroid cytology samples cannot be entirely prevented. Itseffect on smear quality was however minimised by the spontaneous capillary action of new technique as opposed to the active often high suction pressures of conventional FNA procedure. Morphologic details were thus improved resulting in easier interpretation and shorter screening times.

If onlyconventional FNA cytology had been employed 20.60 percent diagnosis would not have been possible because unsuitable sample were obtained for one or two cases out of 495.The corresponding failure rate for non- aspiration cytology was only for 18 cases i.e. 3.63\%. The significance is that out of those one or two cases of unsuitable sample by FNAC the single anaplastic carcinoma was also involved. In these aspects, it was seen that utilization of both procedures rendered the failure rate from 12 percent to 2 percent. So, it is suggested that at least for doubtful cases of getting good sample, that come to 10 to $20 \%$ of FNACs both FNA and NAC sample may be taken so that the failure rate may be reduced $2 \%$.

Samatos and Laiman on studying 50 cases of benign and neoplastic lesion of thyroid found that diagnostic superior slides were obtained in $44 \%$ cases by non-aspiration methods whereas only $8 \%$ by the aspiration method. And the percentage of unsuitable slides for FNAC was $14 \%$ but for NAC it was only $6 \%$. According to them the non-aspiration methods, the main advantage is that the improvement of morphological details to get an easier interpretation within a shorter screening time.

Kumarasinghe and Sheriffdeen sampled 410 thyroid nodules, 127 breast lumps and 43 lymph nodes to compare to FNAC and NAC technique. For the breast lesions, the discomfort felt and the staining with blood were less with the non-aspiration techniques. The adequacy of cellular yield was, however comparable for malignant breast lesions with either technique. For lymph node sampling, both techniques showed equivalent results with regard to the cellularity and the non- aspiration technique was superior in other aspects too. For the thyroid, the non-aspiration techniques were found to be superior tothe aspiration technique in all aspects. However, they concluded that although the overall patient discomfort and blood staining was significantly less, theadequacy of the cellularity was influenced by the anatomical site, the clinical situation, the personal experience and the preference of the operator.

DeyPandRay performed FNA and NA techniques consecutively in 107 lesions from 100 patients. The quality of diagnostic material was assessed using a scoring system based on the cellularity and amount of blood in the smear. The total score in the NA group was significantly higher. $(\mathrm{p}<0.05)$ than that FNA techniques.

Non-aspiration technique was not of much value in cases such as suppurative lesions or thyroglossal cysts. In these cases, diagnostic superior slides were obtained from fine needle aspiration techniques. But no diagnostic superior slides were seen on non-aspiration technique for these lesions. Moreover, two unsuitable slides were there regarding these cases. DeyPand Ray suggested that this procedure cannot be advocated in cystic lesions.Sartos and Leiman are doubtful about the NAC technique with respect to more 
fibrous tumours of lower cellularity, for example breast neoplasms, which evoke a fibrous response in the stroma. DeyPand Ray etal. suggested that this procedure cannot be advocated in cystic bony and fibrous lesions.

Good results with the use of this techniques to sample orbital and periorbital tumours have been reported by Zajdela et al while Brifod et al have used this technique in the follow up of breast carcinoma cases. In lymph nodes, especially in children NA cytology gives an excellent cellular yield. It was found less traumatic and safe in liver and other palpable soft tissue masses.

\section{SUMMARY}

A study was undertaken about the cytodiagnosis of thyroid nodules by fine needle aspiration and nonaspiration technique to do an overall quality assessment of non-aspirationcytology. The study included a fairly representative spectrum of all thyroid lesions and diagnostic accuracy of cytodiagnosis was assessed by comparing theresults with the histological diagnosis.

A total number of 18122 patients came for fine needle aspiration cytology at the Medical College hospital, Kottayam from March 2012 to March 2015 and out of these 3934 patients were with thyroid lesions which comprises $21.71 \%$. At the histopathology laboratory, out of 22,416 specimens received from March 2012 to March 2015, 1451 specimens were thyroid i.e.5\% and of these thyroid lesions $4.7 \%$ proved to be carcinomas. The male female ratio was found to be 1:6

Maximum number of patient belonged to 21 to 40 years $(61.5 \%)$ and minimum 1 to 10 years $(0.07 \%)$.

At the second decade, i.e. during the period of puberty and adolescence the incidences for thyroid lesions were much higher for females compared to that of males (9.4\% for females and $4.6 \%$ for males).

In most of the cases a right sided involvement was seen.

The duration of the swelling varied from 1 month to 38years. The mean duration was 3 years. The maximum incidences of malignant lesions noted were of 1 to 2 years (46\%) and that of benign lesions were above 5 years'duration.

Neoplastic lesions constituted $9.88 \%$ of all thyroid lesions sent for FNAC. As follicular carcinoma, cannot be distinguished cytologically the exact percentage of incidences of carcinoma could not be assessed. Out of 789 cytohistologically correlated thyroid lesions, carcinoma was $5.6 \%$.

The smears taken by aspiration and non- aspiration techniques were stained and interpreted without the knowledge of technique employed for any particular set of slides and found smears by the non-aspiration technique diagnostically much superior than the smears by aspiration technique i.e. $32 \%$ and $4 \%$ respectively. The unsuitable smears were very few (3\%) by non- aspiration technique whereas more for aspiration technique (20\%). The non- aspiration smears were with high cellularity well preserved, unobscured by background blood and excellently displayed with retention of architectural pattern such as follicles, papillae or sheets.

For suppurativelesions, better smears were obtained by aspiration technique, so in such cases both aspiration and non- aspiration techniques may be done.

The introduction of fine needle cytology has a substantial effect on the management of the patient with thyroid swelling. The percentage of patients undergoing thyroidectomy has now been decreased by $25 \%$. Hence it is significant that fine needle cytology is safe, accurate and cost effective and this procedure has a central role in the management of thyroid nodules.

\section{References}

[1]. J., \& L. G. (1988.). Nonaspiration fine needle cytology:Application of a new technique in Nodular Thyroid Diseases. . Acta.Cytologica,32, 352-356.

[2]. Burker, D. F., \& S, P. M. (1956). Fine needle cytology-Is aspiration suction necessary: A study of 100 masses in various sites. Acta. Cytologica, 33, 809-913.

[3]. Stalwart. F. W. (1933). The diagnosis of thyroid tumours by aspiration. Annuals of Surgery, 92, 169.

[4]. Martin M E., \&llisE. E. (1930). Biopsy by needle punctures aspiration. Annuals of Surgery , 92, 169-181.

[5]. Ghosh A, Mishra R K, Sharma S P, Singh H N., \&Chaturvedi A K . 2000). Aspiration vs non-aspiration technique of cytodiagnosis - A Critical evaluation in 160 cases. Indian Journal of Pathology and microbiology, 43, 107-112.

[6]. Haddadi -Nezhad S, Larijani B , Tavangar S M. ,\&Nouraei S M.(2003)Comparative of fine non-needle aspiration with fine needle aspiration technique in cytological studies of thyroid nodule. (2003). Endocrine pathology, 14, 369-373.

[7]. Romitelli F, Di StasioE.,\& Santoro C.(2009). A Comparative study of fine needle aspiration and fine fine needle non-aspiration biopsy on suspected thyroid nodules. (2009). Endocrine pathology, 20, 108-113.

[8]. Modi P, Oza H., \&Bhalodia J. (2014). Utility and adequacy of fine Needle Aspiration Cytology in Head and Neck Lesions: A hospital based study. International journal of scientific studies, 8, 100-105. 